\title{
KIT entwirft agile Produktion für Batteriezellen
}

\author{
Wissenschaftler des KIT entwickeln gemeinsam mit Partnern aus der Wissenschaft ein agiles \\ Produktionssystem. Damit sollen sich Batteriezellen format-, material- und stückzahlflexibel \\ herstellen lassen. Die im Januar gestartete zweite Stufe des Projektes - AgiloBat2 - fördert \\ das Bundesministerium für Bildung und Forschung (BMBF) mit insgesamt 14,5 Millionen Euro.
}

Weltweit steigt die Relevanz der Batterieforschung. Bisher weitgehend unbeachtet blieb dabei die Frage, wie sich Batterien an den verfügbaren Bauraum anpassen lassen, statt wie heute üblich umgekehrt. Eine weitere Herausforderung: Lithium-Ionen-Zellen bestehen unter anderem aus seltenen Elementen, insbesondere Kobalt und Nickel, deren Preise stark schwanken und deren Versorgungslage immer kritischer wird.

„Eine intelligente und nachhaltige Batteriezellproduktion ist entscheidend für die Mobilität der Zukunft, aber auch für Elektronik in unserem heutigen Alltag, wie etwa in Smartphones", sagt der Präsident des KIT, Professor Holger Hanselka. „Im Forschungsprojekt AgiloBat2 arbeiten wir am KIT gemeinsam mit unseren Partnern aus der Wissenschaft daran, dass eine solche Produktion am Wirtschaftsstandort Deutschland möglich wird. Denn mit einem agilen Produktionssystem lassen sich Batteriezellen künftig individuell an die jeweiligen Anfor-

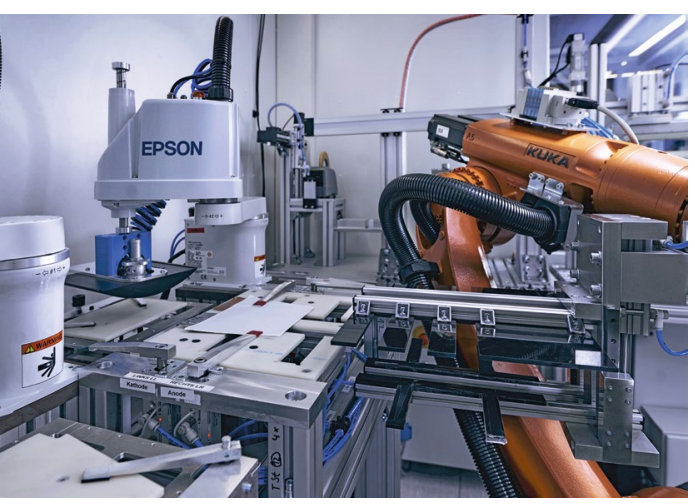

Innenansicht des Produktionssystems mit zentralem Roboter und den drei Produktionsmodulen zum Stapeln (links), Kontaktieren (Mitte) und Verpacken (rechts). (๑ Robert Fuge)

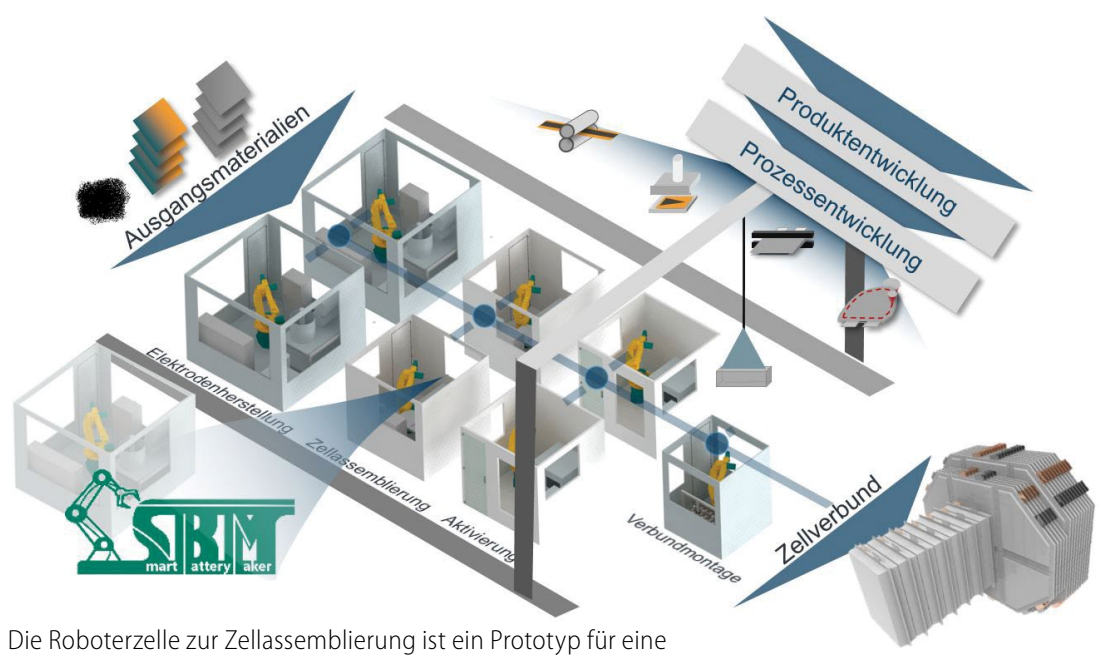

Produktionszelle im Gesamtproduktionssystem AgiloBat. (๔ wbk)

derungen anpassen - und das wird viele neue Möglichkeiten eröffnen."

Vor knapp einem Jahr startete AgiloBatl als reine Entwicklungs- und Konzeptphase im Rahmen des geförderten Innovationscampus Mobilität der Zukunft (ICM). Seit Januar 2021 läuft das zweite geförderte Teilprojekt AgiloBat 2 und damit die Umsetzungsphase. Alle in der Umsetzung gewonnenen Erkenntnisse fließen in das Entwicklungsprojekt zurück und werden dort wieder aufgegriffen.

\section{Karlsruher Forschungsfabrik}

Zunächst erstellt das Forschungsteam ein wandelbares Gesamtanlagendesign. Die Wandelbarkeit des Produktionssystems wird durch den Aufbau eines digitalen Zwillings ermöglicht, der sowohl den Gesamtprozess, als auch Einzelprozesse digital realitätsgetreu abbildet. Somit lässt sich das System entsprechend schnell konfigurieren und mit
Kennzahlen hinterlegen. Parallel dazu wird das Produktionssystem konstruiert. „Hierfür werden wir in der Karlsruher Forschungsfabrik ein agiles Produktionssystem errichten, das sich massiv von allen bisherigen Produktionssystemen im Bereich der Batteriezellfertigung unterscheidet", sagt der Initiator und Leiter der Projekte, Professor Jürgen Fleischer, Leiter des wbk Instituts für Produktionstechnik am KIT. „Es wird skalierbar sein, wodurch es sich von derzeitig etablierten starren Produktionslinien abgrenzt. Dadurch werden wir in der Lage sein, wirtschaftlich kleine bis mittlere Stückzahlen, verschiedene Materialien und Formate abzubilden“. Erreicht werden soll dies durch standardisierte Roboterzellen, die die Produktionsmodule aufnehmen.

Kontakt Karlsruher Institut für Technologie, 76131 Karlsruhe, www.kit.edu 